\title{
The perimeter as a monitor of glaucomatous changes
}

\author{
H. C. DONOVAN, R. A. WEAle, AND CAROL WHEElER \\ From the Institute of Ophthalmology, London, and Moorfields Eye Hospital, City Road, London
}

SUMMARY A small group of glaucoma patients with scotomata were compared with normal coevals. Plots were obtained of the error of each measurement as a function of retinal illumination. A statistical analysis shows the limitations of perimetry as a monitor of the progress of glaucoma. The significance of the data is discussed.

The primary concern of both the glaucomatous patient and his ophthalmologist is that the treatment prescribed should control the condition. A reduction in the intraocular pressure (IOP) to a so-called 'normal' level is only an indication of control, since it has been found that individuals vary in their tolerance to different levels of IOP (Armaly, 1969). It therefore becomes increasingly important to find the most accurate method of determining changes in the visual field.

Sloan (1939) used a modified Ferree-Rand perimeter and introduced static perimetry as a clinical method of examining the visual field. The technique was further advanced by Goldmann (1945) and later by Harms (1950) and Harms and Aulhorn (1959). It is found that static perimetry gives a much greater reproducibility of results (Drance et al., 1967) than does kinetic perimetry. Accordingly we set out to discover within what limits this reproducibility occurs ( $c f$. Verriest and Israel, 1965).

\section{Method}

The 10 patients in this study had a visual acuity better than $6 / 12(0.5)$, good fixation, low refractive errors, and IOPs not greater than $22 \mathrm{mmHg}$ on medication. In order to minimise fatigue only 1 scotomatous eye of each patient was tested. Three normal controls of the same age group were also tested. Each patient underwent 4 sets of tests on the Tübingen Oculus within a few days of one another. The pupil size was recorded.

The IOP of each patient was measured before and after each test. The eye was dilated with phenylephedrine $10 \%$ and cyclopentolate $1 \%$ for 30 minutes. The appropriate reading correction was

Address for reprints: Professor R. A. Weale, Department of Visual Science, Institute of Ophthalmology, Judd Street, London WC1H 9QS then placed in front of the dilated eye and the patient allowed to adapt to the background illumination of 10 asb for 5 minutes (Greve, 1973, p. 30).

A supraliminal $10^{\prime}$ red fixation target was used. The test target was similar in size but white, and the exposure time kept short at 1/10 s (Greve, 1973, p. 86) to eliminate photic waste and vitiating effects of eye movements. The thresholds were measured infra- to supraliminally starting $1^{\circ}$ from the fovea and going out to $30^{\circ}$ in the following way: first the fovea, then the upper nasal, upper temporal, lower nasal, and last the lower temporal meridian at $45^{\circ}$ to the horizontal.

To see whether a learning factor had played a significant part in the results we compared the readings taken on different days and found that the variation of responses was independent of the sequence of visits.

\section{Results}

The threshold of every position along each meridian was measured, but our analysis was confined to points at $5^{\circ}, 10^{\circ}, 15^{\circ}, 20^{\circ}, 25^{\circ}$, and $30^{\circ}$ from the fovea. The 4 readings at each of these points were averaged and the standard deviation was obtained, except when more than one reading at a point exceeded 1000 asb (see Discussion).

Fig. 1 shows the results from 2 eyes, 1 normal and 1 glaucomatous, without reference to retinal meridian. All other eyes tested gave similar results. As can be seen in both graphs, there is clearly a linear relationship between the log standard deviation and log intensity, showing that as the sensitivity of the retina decreases the variability of responses increases almost linearly. In the graph relating to the normal eye the sensitivity decreases, as expected, towards the periphery. The results from the glaucomatous eye, while showing a similarity in this respect, also show that the variability of responses is not linked 


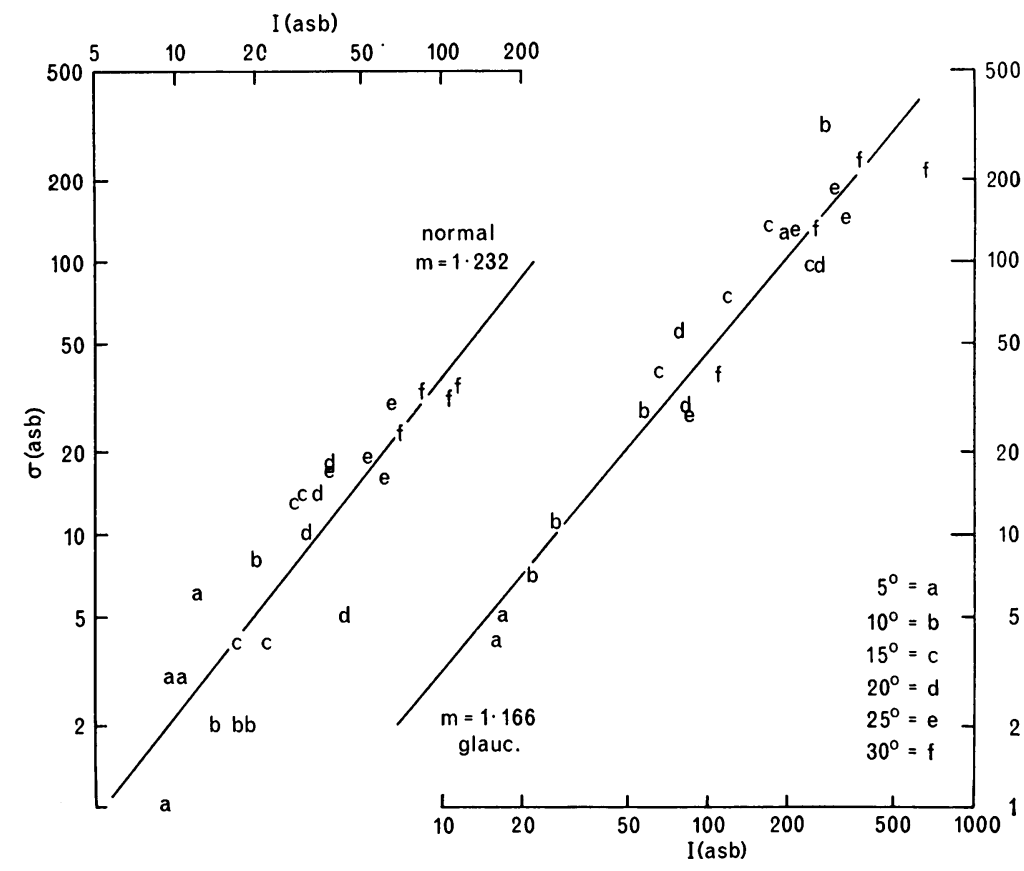

Fig. 1 Abscissa: threshold target luminance. Ordinate: standard error of the measurement $(N=4$, but see text). Both quantities are plotted on log scales. The eccentricities are shown in the inset. The $\mathrm{m}$ values give the slopes of the regression lines. These plots are typical of each of the respective groups of patients

with retinal position but increases wherever the sensitivity is low owing to damage from the glaucomatous process.

The results from all 3 normal eyes also showed positive correlations between eccentricity, threshold, and variance.

\section{Discussion}

We tried to reduce all artefacts to a minimum and to create the best conditions for visual field testing. Despite this the 3 normal eyes tested showed that as the patient's sensitivity decreases towards the periphery, the variability of responses increases, and it is probable that, had readings been taken beyond $30^{\circ}$, any likely functional change would not have been distinguishable from one due just to chance. The results from the glaucomatous eyes also suggest that it was impossible to predict visual field changes using this method for damaged parts of the retina.

Our experiments highlighted the interesting fact that the variability of the patients' responses appeared to be directly related to the sensitivity of the retina, and where the retina was damaged the variability was greatest. This may have a significant bearing on the view that in some cases medication can reduce the size of the defect, and it should make us very wary of all but the grossest change in the defective visual field. The data are sufficiently detailed to enable one to scrutinise them quantitatively.

As any perimetric measurement involves the determination of a visual threshold, clinical tests will be subject at least to the variables affecting such measurements in the laboratory. It is not feasible to subject patients to the rigours of experimental control, and this means that their thresholds will almost certainly be higher than those measured under optimum conditions. In addition we used a nonzero background luminance, which also raises the threshold, the amount varying with the pupil area when this is the limiting aperture. However, both our normal thresholds and their standard deviations are similar to the results obtained by Verriest and Israel (1965) on another instrument, namely, the Goldmann perimeter, and this lends confidence to the belief that our normal observers deserve this epithet.

A variety of theories has been propounded to explain the influence of various parameters on the visual threshold. The best known is due to Hecht et al. (1942), who based their interpretation on the quantum nature of light. They argued that weak flashes contain an indeterminable (small) number of light quanta and that the eye will signal 'seen' when a given flash contains a minimum (mean threshold, $a$ ) number of quanta or more. In such a situation it is possible to relate the probability, $\boldsymbol{P}$, seeing a flash of mean intensity, $m$ (absorbed by the 
Fig. 2 The logarithm of the variance (var) as a function of the mean threshold I (in asb). Note the constant value of the variance above I $\sim 250$ asb. The zero value for log variances at the extreme ends of the range represent var $=0$. The low value at the upper limit is spurious, and explained in terms of the instrument being unable to deliver more than 1000 asb. (Age spans: Normal, 55 to 62 years; patients, 56 to 62 years)

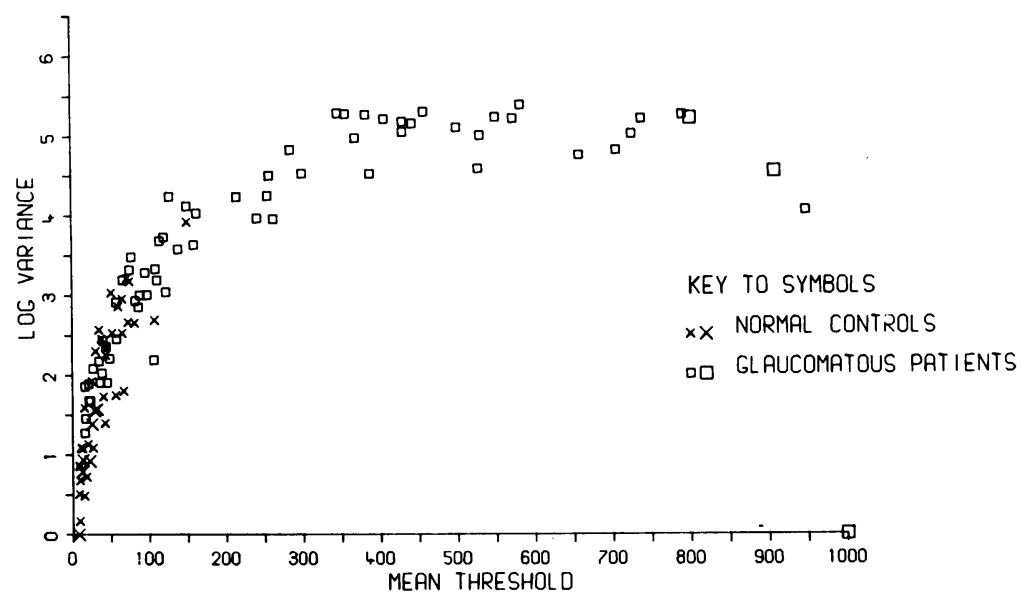

retina), when the actual number of quanta is $i$. It can be shown that

$$
P=1-\sum_{i=0}^{n-1} e^{-a}\left(a^{i} / i !\right)
$$

The relevance of this expression to our results is as follows. The equation is based on the Poisson distribution, a characteristic of which is that the variance and mean are equal. It can be shown that, for the lowest thresholds of our normal observers, this condition is approached. For example, a log mean of 0.92 is associated with a standard deviation, $\sigma$, of $0 \cdot 1 \log$ units. But $\sigma^{2}=\operatorname{var} /(n-1)$, where $n$ is the number of observations. Thus var $=4.8 \mathrm{asb}$, which is of the same order as the mean (antilog of $0.92)=8.3 \mathrm{asb}$. A study of the variance is consequently sometimes helpful in selecting one of several possibilities as regards underlying mechanisms.

It should be noted that the data do not obey the predicted relation accurately, and attention is drawn to the fact that the way in which even the normal 'patient' responds to the situation makes this unlikely. In the rigorous tests the probability of seeing a given stimulus is derived from a large number (about 50) presentations. This is impossible in a clinic. In our study the light intensity was increased till the patient said he could see it. We show in the Appendix that this well-known technique makes it theoretically difficult to use it quantitatively to set confidence limits of such narrowness as to enable one to monitor the progress of, or recovery from, retinal disease by its means.

However, the data obtained on the glaucomatous patients lead to a startling conclusion, which is easy to explain on a theoretical basis.

Fig. 1 indicates the large standard deviations recorded by the patients for the defective parts of their visual fields. In some retinal regions the results are so varied as to appear to be purely random and to come from a uniform distribution. It can be shown that, if the readings are made on a continuous scale, the variance of such a distribution is $C^{2} / 12$ where $C$ is the range of possible readings, that is, the gamut of the instrument. If we assume $C$ is 1000 (classing readings greater than the intensity which the instrument can deliver as 1000 , which will tend to lower the variance), then the variance for each estimate is $1000^{2} \div 12=83333$, the log of which is 4.92. As shown in Fig. 2, there is close agreement between this predicted and the observed values.

This plot shows that the prediction is fulfilled for 250 apostilbs and above, which is not more than a factor of 10 above likely normal thresholds. We interpret Fig. 2 as meaning that, if a blind person were seated at the perimeter, a similar result would be obtained. This approach provides a clue to its cause: it is likely that the patients were guessing. It is also possible that, because of the smallness of the target, minute eye movements led to a patchy retina, giving rise to a large variation.

Whatever the ultimate explanation of the uniform distribution of readings which we have observed, if our observations are typical they rule out the possibility of obtaining useful confidence limits for those retinal regions for which the mean threshold exceeds about 250 asb. Thus, while perimetry has a diagnostic value in these circumstances, its usefulness as a monitoring device can be overrated.

\section{Appendix}

Assume that the intensity of a light flash is increased till the patient signals 'seen'.

The amount of energy at the $j^{\text {th }}$ flash, $j=0,1,2 \ldots$ 
$k$ is fixed as $10^{j / 10}$ apostilbs, where $10^{k / 10}$ is the last measurable amount of energy ( $k=30$ in this case).

If $X_{j}=1$ when the $j^{\text {th }}$ flash is seen and 0 otherwise then

$$
P\left(T=m_{t}\right)=\left(\prod_{j=0}^{t-1} p\left(X_{j}=0\right) \cdot P\left(X_{t}=1\right)\right.
$$

where $T$ is the recorded threshold and $m_{t}$ is the energy flashed in experiment, $t$.

If the number of quanta incident on the cornea at flash $j$ is denoted by $m_{j}$, then $p\left(X_{j}=0\right)=P$ (fewer than $n$ quanta absorbed out of a possible $m_{j}$ ) where $n$ is the number of quanta required for vision.

Assuming Bernoulli trials, that is, that there are two outcomes: either the quantum is absorbed by the retina, with probability, $P$, or it is not, with probability $1-P$.

$$
P\left(X_{j}=0\right)=\sum_{r=0}^{n-1} m_{j} C_{r} \cdot p^{r}(1-p)^{m_{j}-r}
$$

Therefore

$$
\begin{aligned}
P\left(T=m_{t}\right)= & \prod_{j=0}^{t-1}\left[\sum_{r=0}^{n-1} m_{j} C_{r} p^{r}(1-p)^{m_{j}-r}\right] \times \\
& {\left[\sum_{r=n}^{m_{t}} m_{t} C_{r} \cdot p^{r} \cdot(1-p)^{m_{t}-r}\right] }
\end{aligned}
$$

The mean and variance of this complex probability distribution are not known but it is included in the paper to emphasise the difficulty of using this particular method of perimetry to set confidence limits for future readings on the same area of retina of the same patient. Because of this it is difficult to determine whether the patient's threshold has increased or whether the higher threshold is a purely random variation.

Approximate confidence limits can be calculated for normal retinal parts of patients by first finding the mean and variance for the logged data (this transformation is done to remove the skewness from the data introduced by the fact that the amount of energy was increased exponentially and not linearly), and calculating the confidence intervals as approximately

$$
\bar{X} \pm 1.96 s
$$

where $\bar{X}$ is the mean of the logged data and $s$ is the square root of the variance of the logged data. To obtain confidence intervals for the original data, take the antilog of the two values; $95 \%$ of future readings should lie within these limits. For example, the readings $40,50,50,100$ give a range of between approximately 28 and 123 , and the readings $7 \cdot 9,7 \cdot 9,10,10$ give a range of between 6.8 and 11.6 .

We are indebted to Mr Redmond Smith for permission to study his patients. We thank the H. C. Binstead Memorial Fund for financial assistance.

\section{References}

Armaly, M. F. (1969). The visual field defect and ocular pressure level in open angle glaucoma. Investigative Ophthalmology, 8, 105-124.

Drance, S. M., Wheeler, C., and Pattullo, M. (1967). The use of static perimetry in the early detection of glaucoma. Canadian Journal of Ophthalmology, 2, 249-258.

Greve, E. L. (1973). Single and Multiple Stimulus Static Perimetry in Glaucoma; the Two Phases of Visual Field Examination, pp. 30 and 86. Junk: The Hague.

Goldmann, H. (1945). Grundlagen exakter Perimetrie. Ophthalmologica, 109, 57-70.

Harms, H. (1950). Entwicklungsmöglichkeiten der Perimetrie. Albrecht von Graefes Archiv für Ophthalmologie, 150, 28-57.

Harms, H., and Aulhorn, E. (1959). Vergleichende Untersuchungen über den Wert der quantitativen Perimetrie, Skiaskotometrie und Verschmelzungsfrequenz für die Erkennung beginnender Gesichtsfeldstörungen beim Glaukom. Documenta Ophthalmologica, 13, 303-332.

Hecht, S., Shlaer, S., and Pirenne, M. H. (1942). Energy, quanta and vision. Journal of General Physiology, 25, 819-840.

Sloan, L. (1939). Instruments and techniques for the clinical testing of light senses III. An apparatus for studying regional differences in light sense. Archives of Ophthalmology, 22, 233-251.

Verriest, G., and Israel, A. (1965). Application du périmètre statique de Goldmann au relève topografique de seuils différentiels de luminance pour de petits objects colorés projetés sur un fond blanc. Vision Research, 5, (151-174. 